\title{
Detection and parenteral treatment of iron deficiency in anemia of major burns.
}

\author{
Carbajal-Guerrero Ja, Gacto-Sánchez Pa, López-Chozas JMb. \\ aBurn Unit. Hospital Virgen del Rocío. Sevilla. Spain \\ b Internal Medicine department. Hospital Virgen del Rocío. Sevilla. Spain
}

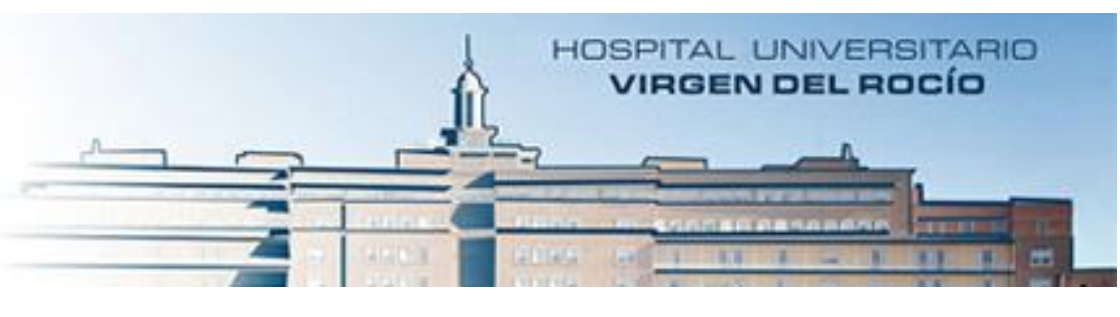

\section{Introduction}

Classically, anemia of burns has been considered a combination of blood loss and anemia of critical illness. Blood loss is considered a relevant etiology of iron deficiency and anemia. Recent studies establish that $17 \%$ of all units are transfused after last surgery in burns ${ }^{1}$. Deleterious effects of transfusions are widely accepted, and confirmed in burn population?

In our everyday clinic assistance progressive anemia once surgical phase was finished was observed in some patients. Progressive anemia led patients to new transfusions although bleeding was not detected. This kind of anemia was presumed to be related with critical illness. But we wonder whether iron deficiency could play a role in this progression.

\section{Results}

23 patients underwent surgery at least twice during the mentioned period. Twelve of these patients showed progressive anemia once the surgery phase had finished. Anemia was rapidly progressive requiring blood transfusion (haemoglobin $<8 \mathrm{~g} / \mathrm{dl}$ ) before iron metabolism could be evaluated in two of them. Iron metabolism test in the other 10 suggested iron defficiency. Two of them were not considered for parenteral iron replacement (extremely high levels of ferritin, active severe sepsis).

Finally, eight patients were considered for iron intravenous replacement with ferric carboxymaltose. No transfusions were needed in these patients after iron administration.

\begin{tabular}{|c|c|c|c|c|c|c|c|c|c|c|c|c|}
\hline Age & BSA & $\begin{array}{c}\text { Full } \\
\text { Thickness }\end{array}$ & $\begin{array}{c}\text { Escarecth } \\
\text { omies }\end{array}$ & $\begin{array}{l}\text { Blood } \\
\text { units } \\
\text { periop }\end{array}$ & $\begin{array}{l}\text { Blood } \\
\text { units } \\
\text { postop }\end{array}$ & $\begin{array}{l}\text { Hb pre- } \\
\text { iron }\end{array}$ & $\begin{array}{l}\text { Hb post- } \\
\text { iron }\end{array}$ & $\begin{array}{l}\text { Serum } \\
\text { iron }\end{array}$ & $\begin{array}{c}\text { iron- } \\
\text { binding } \\
\text { capacity }\end{array}$ & $\begin{array}{l}\text { transferrin } \\
\text { saturation. }\end{array}$ & Ferritin & STfR \\
\hline 35 & 49 & 42 & 11 & 68 & 0 & 8.0 & 8,9 & 24 & 122 & 19 & 250 & \\
\hline 75 & 16 & 16 & 3 & 4 & 0 & 8.7 & 9.8 & 26 & 183 & 14 & 152 & \\
\hline 27 & 40 & 15 & 3 & 6 & 0 & 7.5 & 9.5 & 51 & 283 & 18 & 835 & 3.3 \\
\hline 77 & 18 & 9 & 3 & 8 & 0 & 8.1 & 9.6 & 16 & 205 & 08 & 291 & 1.4 \\
\hline 44 & 24 & 16 & 8 & 6 & 0 & 9.2 & 10.8 & 19 & 164 & 12 & 148 & 3.2 \\
\hline 29 & 27 & 27 & 2 & 2 & 0 & 8.2 & 10.1 & 32 & 246 & 13 & 472 & 3.7 \\
\hline 44 & 20 & 20 & 1 & 1 & 0 & 7.8 & 8.6 & 19 & 147 & 13 & 152 & 3.5 \\
\hline 42 & 45 & 35 & 5 & 35 & 0 & 8.2 & 8.8 & 28 & 194 & 14 & 331 & 3.9 \\
\hline 31 & 70 & 70 & 12 & 37 & 2 & 9.4 & & 37 & 210 & 18 & 922 & 2.4 \\
\hline 52 & 18 & 18 & 5 & 12 & 0 & 9.5 & & 29 & 224 & 13 & 310 & 2.6 \\
\hline 24 & 18 & 9 & 2 & 1 & 2 & 9.5 & & 42 & 319 & 13 & 56 & 3.3 \\
\hline 55 & 45 & 40 & 7 & 28 & 2 & 8.8 & & 23 & 64 & 35 & 1.193 & 1.6 \\
\hline
\end{tabular}

\section{Methods}

Between february 2016 and december 2016, patients undergoing surgery at least twice in our burn unit recibed spacial tracking. We followed haemoglobin progression once surgical phase was finished (48h after last surgery and afterwards). If haemoglobin falled below $\leq 9.5 \mathrm{~g} / \mathrm{dl}$, and no bleeding source was detected, an iron metabolism study was carried on in order to detect iron defficiency. As serum ferritin was frequently elevated, measurement of serum soluble transferrin receptors (STfR) was added later on, with the purpose of trying to differentiate anemia of critical illness from iron deficiency. If iron deficiency was suspected, an adjusted dose of ferric carboxymaltose was intravenously administered.

\section{Conclussions}

Iron defficiency was detected after surgical phase in almost $50 \%$ of the patients undergoing surgery at least twice. Ferritin was elevated in almost all patients as an acute phase-reactant and was unhelpfull to detect iron deficiency. StfR was not able to detect iron defficiency as accurately as expected, probably because standardization of assays are not yet complete ${ }^{3}$. However, we evaluate that stopping anemia progression and avoiding new transfussions after iron administration in our patients could be considered a clear clinical response to the treatment.

Iron defficiency should be evaluated when progressive anemia affects burn patients without active bleeding.

1) Posluszny JA, Jr., Conrad P, Halerz M, Shankar R, Gamelli RL. Classifying transfusions related to the anemia of critical illness in burn patients. J Trauma 2011 Jul;71(1):26-31

(2) Palmieri TL, Caruso DM, Foster KN, Cairns BA, Peck MD, Gamelli RL, et al. Effect of blood transfusion on outcome after major burn injury: a multicenter study. Crit Care Med 2006 Jun;34(6):1602-7

(3) Lopez A, Cacoub P, Macdougall IC, Peyrin-Biroulet L. Iron deficiency anaemia. Lancet 2016 Feb 27;387(10021):907-16. 